\title{
Coherent control of acoustic phonons in semiconductor superlattices
}

\author{
Albrecht Bartels, ${ }^{\text {a) }}$ Thomas Dekorsy, and Heinrich Kurz \\ Institut für Halbleitertechnik, Rheinisch-Westfälische Technische Hochschule Aachen, Sommerfeldstr. 24, \\ D-52056 Aachen, Germany \\ Klaus Köhler \\ Fraunhofer-Institut für Angewandte Festkörperphysik, D-79108 Freiburg, Germany
}

(Received 5 January 1998; accepted for publication 27 March 1998)

\begin{abstract}
Coherent acoustic phonons are generated in GaAs/AlAs superlattices by excitation with femtosecond laser pulses. Several modes of the acoustic phonon spectrum are observed, in agreement with the effect of zone folding in the mini-Brillouin zone of the superlattice. By applying successive pump pulses we are able to silence the first back-folded mode near $q=0$, while selectively enhancing the coherent amplitude of higher order backfolded modes. This increase in the spectroscopic sensitivity opens the way to detailed time resolved studies of higher order acoustic modes in superlattices.
\end{abstract}

The coherent control of elementary optical excitations is one of the key issues in femtosecond technology. This control is enabled by the complete knowledge of the dynamics of quantum mechanical systems concerning amplitude and phase in the time domain. Thus, providing well defined quanta of energy in a well defined temporal sequence a system can be driven into an artificial quantum mechanical state, which cannot be achieved by other means of optical excitation. Presently, strong endeavors are made to coherently control electronic and vibronic excitations in solids, as well as in chemical and biological systems. Coherent control over vibronic excitations has been accomplished in molecular systems, where the amplitude of molecular vibrations was enhanced extremely by exciting the system with a comb of laser pulses spaced by the inverse of the vibrational period. ${ }^{1}$ In photochemistry, coherent control is pursued in order to sequentially excite certain reaction paths, which would be very improbable in thermodynamic equilibrium. ${ }^{2}$ In semiconductor heterostructures, the coherent control of electronic excitations has been demonstrated convincingly., ${ }^{3,4}$ The phase coherence of excitons was used to excite and destruct excitons, thus controlling the carrier population ${ }^{4}$ and the emission of $\mathrm{THz}$ radiation ${ }^{3}$ on a femtosecond time scale. The coherent control of lattice oscillations in solid state materials is an intriguing concept, since the selective manipulation of the amplitude of certain lattice modes allows a detailed study of electron-phonon and phonon-phonon interaction, as well as anharmonicity at large amplitudes. So far, coherent control of LO phonons has been realized in bulk semiconductors in double-pulse experiments, where the careful choice of the excitation parameters led to a complete destructive interference of coherent LO phonons. ${ }^{5,6}$ Phase control over degenerate phonon modes has also been achieved recently. ${ }^{7}$ Here, we present the first demonstration of coherent control over acoustic phonons in semiconductor superlattices, which, in principle, allows to selectively excite any branch in the phonon dispersion, while suppressing other branches.

In the lower frequency range acoustic phonons exhibit a

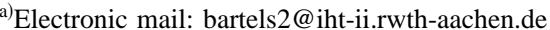

linear dispersion relation $\omega=v_{\text {sound }} q$, where $v_{\text {sound }}$ is the sound velocity of the material and $q$ the wave vector of the phonon. In artificial superlattices, the acoustic phonon spectrum exhibits zone-folding within the mini-Brillouinzone due to the new periodicity along the growth direction. This leads to the appearance of several acoustic phonon modes with $\omega$ $\neq 0$ at $q \approx 0,{ }^{8}$ which are accessible by light scattering experiments. The dispersion relation in the lowest order approximation ${ }^{9}$ is

$$
\omega_{p, q}^{2}=\frac{C_{0}}{\rho_{0}} \times(q+p G)^{2},
$$

where $C_{0}$ and $\rho_{0}$ are the zeroth Fourier expansion coefficients of the elastic stiffness constant $C_{11}$ and the crystal density, respectively, expanded along the growth direction. $G=2 \pi /\left(d_{\mathrm{GaAs}}+d_{\mathrm{AlAs}}\right)$ is the mini-Brillouin zone boundary. $p$ takes the values $p=0, \pm 1, \pm 2, \ldots$ and $|p|$ denotes the order of the phonon mode. Solutions to Eq. (1) are degenerate modes at $q=0$ with $\omega=\left(C_{0} / \rho_{0}\right)^{1 / 2}|p| G$.

Femtosecond time resolved investigations are performed in a standard pump probe setup. The acoustic lattice vibrations are excited at room temperature resonantly with the E1HH1 transition of the superlattice by an intense pump pulse derived from a tunable modelocked Ti:Sapphire laser focused to a spot of $\approx 40 \mu \mathrm{m}$ on the sample. The pulse duration of $50 \mathrm{fs}$ is far below the period $2 \pi \omega^{-1}$ of the first and second order folded acoustic phonons $(>1 \mathrm{ps})$ resulting in the impulsive generation of coherent acoustic lattice vibrations. ${ }^{10}$ In order to exercise coherent control over the lattice motion a Michelson interferometer is inserted into the pump beam. The temporal spacing between the two resulting pump pulses is adjusted by the interferometer mirrors. It is measured via correlation with the probe pulse using twophoton absorption in GaP and can be realized with an accuracy of $\approx 10$ fs. The intensities of the two pump pulses are adjusted with neutral density filters.

A probe pulse of lower intensity, detected with a $\mathrm{Si}$ $p-i-n$ diode after reflection from the sample, is used to observe relative reflectivity changes at the energy of the first interband transition as a function of the time delay between 


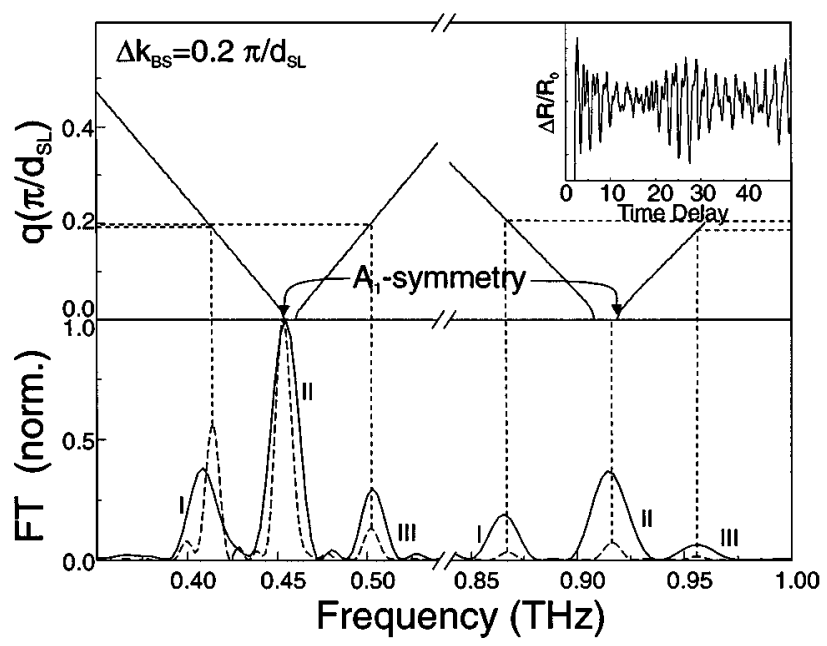

FIG. 1. Calculated dispersion (top) and normalized Fourier spectrum (bottom) of the oscillating component of the transient reflectivity change after single-pulse (dashed) and double-pulse excitation (solid line) of sample A. The transient reflectivity change for double-pulse excitation is shown in the inset.

pump and probe pulse. Oscillating components of the reflectivity changes are induced by the coherent lattice vibrations via refractive index changes due to the acoustic deformation potential. ${ }^{11}$ The transient reflectivity signature is dominated by electron-hole pairs generated under resonant conditions. An initial reflectivity change $\Delta R / R_{0}$ at the first interband transition in the order of $10^{-3}$ is induced. The slow decay of the free carrier density with a time constant of some $100 \mathrm{ps}$ is modulated by the signature of the coherently generated acoustic phonons with an amplitude of $\approx 10^{-5}$. In order to separate the lattice dynamics from the carrier relaxation the oscillating signature is extracted numerically.

Two different superlattices are investigated. Sample A consists of 40 periods of 19 monolayers (ML) of GaAs and 19 ML of AlAs grown on (001) oriented GaAs substrate. Sample B contains 16 ML GaAs and 16 ML AlAs. Figure 1 depicts the Fourier transform of the time domain data obtained in sample A with single-pulse and double-pulse excitation with pulses of equal intensity and the dispersion calculated with a model treating the superlattice as an elastic continuum according to Rytov. ${ }^{12}$ The single-pulse spectrum reveals a strong triplet of peaks around $450 \mathrm{GHz}$ and a second weaker triplet around $900 \mathrm{GHz}$, which are assigned to the first and second order backfolded modes. The peaks labeled II correspond exactly to the calculated $q=0$ phonon frequency of $A_{1}$ symmetry, while the peaks I and III are in excellent agreement with the frequencies at $q=2 k_{\text {laser }}$ of the calculated dispersion. The excitation of these modes is attributed to forward (peak II) and backward (peaks I and III) scattering. The selection rules for Raman scattering ${ }^{9}$ prevent the observation of four frequencies (two doublets at $q=0$ and $q=2 k_{\text {laser }}$ ), since one $q=0$ mode of each order (upper branch of first order and lower branch of second order for sample A) is symmetry forbidden. Furthermore, the observed relative peak intensities within one triplet agree with calculated cw-scattering intensities. ${ }^{9}$ This strongly points towards resonant impulsive stimulated Raman scattering as excitation mechanism. $^{11}$

Double-pulse excitation is performed in order to con- a)

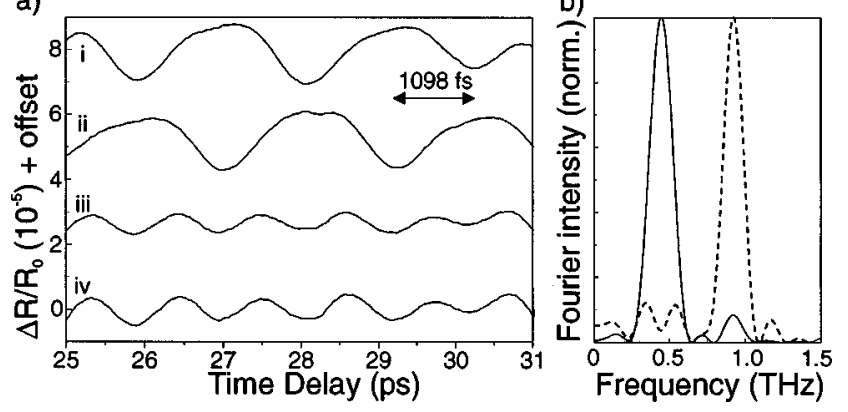

FIG. 2. (a) Transient reflectivity changes at a time delay of $25 \mathrm{ps}$ induced by the first pulse (curve i), second pulse (curve ii) and both pulses (curve iii) in sample A. Curve iv depicts the transient obtained from a numerical superposition of curves i and ii. (b) Spectra of curve i (solid line) and curve iii (dashed)

structively interfere two sound waves of second order and simultaneously destructively interfere two first order waves. Two pump pulses of equal intensity are separated in time by half the period of the $q=0$ mode of a first order triplet, i.e., 1098 fs for sample A. Two restrictions on the feasibility of the dual-excitation experiment have to be taken into account. The first one concerns the dephasing of the coherent phonons within the delay between the two pump pulses. This does not present a problem, since the dephasing time constants are in the range of $100 \mathrm{ps},{ }^{10}$ which is large compared to the delay time. The second requirement concerns the saturation of the interband absorption by the pump pulses. A pump power of $50 \mathrm{~mW}$ per beam should not be exceeded, because power dependent measurements revealed the saturation of the coherent acoustic amplitude above $100 \mathrm{~mW}$, corresponding to the saturation of the interband absorption. Therefore $40 \mathrm{~mW}$ per pump pulse are chosen in the double pulse experiment.

The transient reflectivity change induced by the lattice vibrations of this experiment is depicted in the inset of Fig. 1. The signature of the amplified second order vibrations can be identified in the time domain data though the destructive interference of the first order is not fully accomplished. The normalized Fourier spectrum (Fig. 1) clearly shows that the relative intensities of the second order to first order peaks are enhanced by a factor of 5 compared to the single-pulse excitation. The incomplete extinction of the first order mode is attributed to a rough adjustment of the pulses to equal intensities. A partial bleaching of the interband transitions by pulse No. 1 results in a decreased driving force exerted by pulse No. 2.

Total extinction of the first order vibrations is achieved by careful adjustment of the pump powers. The static time delay of the probe pulse is set on the beat maximum of the coherent vibration around 25 ps. Figure 2(a) shows the time domain data for the first pulse only (curve i), the second pulse only (curve ii) and both excitation pulses present (curve iii). No more first order vibration can be identified in curve iii, neither from the transient nor from the Fourier spectrum, which is shown in Fig. 2(b) in comparison to a single pulse excitation. The linewidths are broader than those in Fig. 1 due the the small temporal window of 5 ps, thus the triplets observed in Fig. 1 are not resolved. Curve iv shows the result of the addition of curves $i$ and ii. The result is 


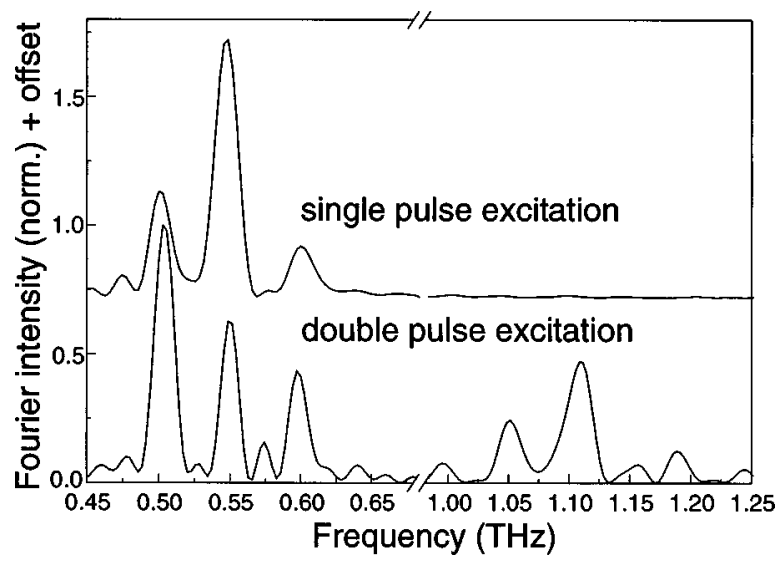

FIG. 3. Normalized Fourier spectrum of the double-pulse excitation in sample B compared to the single-pulse excitation.

hardly distinguished from the result obtained by the two pulse excitation, demonstrating the successful suppression of the first order mode by destructive interference of two independently generated sound waves in the experiment.

In sample A the second order phonon modes could already be resolved directly in the single-pulse excitation configuration, which is not the case for sample B. This discrepancy is attributed to a poorer sample quality of sample B, as confirmed by a smaller signal-to-noise ratio in comparison to the experiments on sample A. The experimental conditions for the dual excitation of sample B are the same as for sample A except for the temporal spacing of the pump pulses being $910 \mathrm{fs}$ corresponding to half the period of the first order backfolded mode and the laser energy tuned to the E1HH1 transition $(1.6 \mathrm{eV})$. The Fourier intensity spectrum is shown in Fig. 3. Total extinction of the first order oscillations is not achieved. The second order triplet can now be resolved at the expected frequency position. It is very important to resolve the second order vibrations in order to clarify the physical origin of the observed triplets. For example, fluctuations of the superlattice period could produce multiple frequencies with a spacing that is doubled in second order. This does not happen if the reason for the triplet structure is resonant stimulated Raman scattering in forward and backward scattering geometry. Furthermore, the width of the forbidden frequency bands in the acoustic dispersion can be measured in the time resolved technique. These splittings in the dispersion and the relative peak intensities are sensitive towards the layer interfaces and can provide information on interface disorder in the sample. ${ }^{13}$

We did not succeed in the observation of third order vibrations by applying three pump pulses, since the losses in two Michelson interferometers resulted in a maximum power of only $10 \mathrm{~mW}$ per beam and the coupling of third order modes to light is presumably very low. ${ }^{9}$

In conclusion, we demonstrated the coherent control of zone-folded acoustic lattice vibrations in GaAs/AlAs superlattices by time sequential excitation with fs pulses. First order modes can efficiently be silenced while simultaneously enhancing the second order vibrations. Thus we have shown, in principle, that it is possible to selectively drive one specific elementary excitation of a crystal while suppressing undesired modes. This allows the preparation of the phonon system in a state not achievable in thermal equilibrium, continuous wave or single-pulse coherent excitation. Furthermore, we showed that one can use double-pulse excitation in order to observe modes, which are not resolved in a singlepulse experiment because of lower signal-to-noise ratio. Besides the general interest in controlling elementary excitations in solids these experiments open the way to systematic investigations of dephasing times of first and second order acoustic phonons. These dephasing times are of great interest in theoretical considerations on the acoustic phonon bottleneck in the carrier relaxation in semiconductor heterostructures. Applications in material or medical science are feasible by generating highest frequency sound waves with large amplitudes by multiple pulse excitation by pulseshaping techniques. ${ }^{1}$

This work is supported by the Deutsche Forschungsgemeinschaft (Ku 540/15-2).

${ }^{1}$ A. M. Weiner, D. Leaird, G. P. Wiederrecht, and K. A. Nelson, J. Opt. Soc. Am. B 8, 1264 (1991).

${ }^{2}$ W. S. Warren, H. Rabitz, and M. Dahleh, Science 259, 1581 (1993).

${ }^{3}$ P. Planken, I. Brener, M. Nuss, M. Luo, and S. L. Chuang, Phys. Rev. B 48, 4903 (1993).

${ }^{4}$ A. P. Heberle, J. J. Baumberg, and K. Köhler, Phys. Rev. Lett. 75, 2598 (1995).

${ }^{5}$ T. Dekorsy, W. Kütt, T. Pfeifer, and H. Kurz, Europhys. Lett. 23, 223 (1993).

${ }^{6}$ M. Hase, K. Mizoguchi, H. Harima, S. Nakashima, M. Tani, K. Sakai, and M. Hangyo, Appl. Phys. Lett. 69, 2474 (1996).

${ }^{7}$ C. J. Brennan and K. A. Nelson, J. Chem. Phys. 107, 9691 (1997).

${ }^{8}$ P. Y. Yu and M. Cardona, Fundamentals of Semiconductors (Springer, Berlin, 1996).

${ }^{9}$ B. Jusserand, D. Paquet, F. Mollot, F. Alexandre, and G. Le Roux, Phys. Rev. B 35, 2808 (1987).

${ }^{10}$ A. Yamamoto, T. Mishina, Y. Masumoto, and M. Nakayama, Phys. Rev. Lett. 73, 740 (1994); K. Mizoguchi, K. Matsutani, S. Nakachima, T. Dekorsy, H. Kurz, M. Nakayama, Phys. Rev. B 55, 9336 (1997).

${ }^{11}$ A. Bartels, T. Dekorsy, and H. Kurz (unpublished).

${ }^{12}$ S. M. Rytov, Acoust. Zh. 71, 2 (1956).

${ }^{13}$ B. Jusserand and M. Cardona, Light Scattering in Solids V (Springer, Berlin, 1989). 